\title{
Diseño in silico de quinolonas con nanoacarreador de $\mathrm{TiO}_{2}$
}

\section{In silico design of quinolones with $\mathrm{TiO} 2$ nano-carrier}

\author{
A. Albino-Flores ${ }^{\text {(D) }}$, E. P. García-Tejada ${ }^{\text {a }}$, L. Fuentes-Ocampo ${ }^{\text {b }}$, E. Diaz-Cervantes $\left(D^{a}, *\right.$ \\ ${ }^{a}$ Departamento de Alimentos, Centro Interdisciplinario del Noreste (CINUG), Universidad de Guanajuato, 37975 Tierra Blanca, Guanajuato, México. \\ ${ }^{b}$ Departamento de Enfermería y Obstetricia, Centro Interdisciplinario del Noreste (CINUG), Universidad de Guanajuato, 37975 Tierra Blanca, Guanajuato, México.
}

\begin{abstract}
Resumen
En este trabajo se realizó el diseño in silico de 17 moléculas basadas en la estructura de una quinolona, en las que se evaluó el efecto de triazoles, tetrazoles e isatinas como grupos funcionales a través del aumento de la concentración mínima inhibitoria (MIC, por sus siglas en inglés) calculado mediante un estudio de relación cuantitativa de estructura-actividad (QSAR, por sus siglas en inglés) haciendo comparativa con el MIC experimental de quinolonas comerciales. Además, se realizaron acoplamientos moleculares con el ADN-topoisomerasa IV de S. aureus (blanco biológico) para conocer el sitio y energía de acoplamiento al blanco biológico de quinolonas. A partir de estos resultados se realizaron complejos entre las mejores quinolonas comerciales y estructuras diseñadas unidas a una celda unitaria de $\mathrm{TiO}_{2}$, fungiendo como nano-acarreador para la liberación del fármaco.
\end{abstract}

Palabras Clave:

Quinolonas, Diseño in silico, $\mathrm{TiO}_{2}$, Nano-acarreador, QSAR, S. aureus.

\begin{abstract}
In this work, the in silico design of 17 molecules based on the structure of a quinolone was carried out, which the effect of triazoles, tetrazoles and isatins as substituents was evaluated considering the increase of the minimum inhibitory concentration (MIC) calculated through a quantitative structure-activity relationship (QSAR) study comparing with the experimental MIC of commercial quinolones. In addition, molecular couplings were performed with $S$. aureus DNA topoisomerase IV (biological target) to determine the site and energy of coupling to the biological target of quinolones. From these results, complexes were made between the best commercial

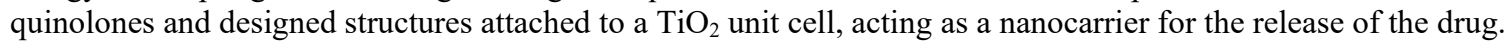

Keywords:

Quinolones, in silico design, $\mathrm{TiO}_{2}$, Nano-carrier, QSAR, S. aureus.

\section{Introducción}

Las infecciones nosocomiales son aquellas infecciones adquiridas en hospitales y otros centros sanitarios, generalmente son causadas por bacterias, y diseminadas a través de herramientas médicas, superficies o alimentos contaminados (López-Cerero, 2014). Afectan entre un 5 y 10\% de los pacientes que ingresan a hospitales modernos (Mazón \& Verneiulle, 2019); son una de las principales causas de morbilidad y mortalidad a nivel global; prolongan la hospitalización, generando costos adicionales en el tratamiento del paciente (Lebeque et al., 2006). Se presentan frecuentemente en personas con condición inmunosuprimida, ya sea por enfermedades adyacentes, quimioterapias o edad avanzada (Maguiña, 2016).
Este problema se agrava cuando las bacterias adquieren resistencia a antibióticos, causando enfermedades más severas que requieren medicamentos más agresivos para controlar la infección, lo que a su vez aumenta los efectos adversos ocasionados por el fármaco. El uso indiscriminado de antibióticos ha permitido a las bacterias desarrollar nuevos mecanismos de resistencia rápidamente (Pachay, 2018). Se estiman 700,000 muertes en 2016 debido a bacterias resistentes y se prevé que la cifra podría ascender a 10 millones en el año 2050 si no cambia la tendencia (Oteo, 2019).

\footnotetext{
*Autor para la correspondencia: e.diaz@ugto.mx 1.fuentes@ugto.mx (Lucero Fuentes-Ocampo).

Correo electrónico: 15124@utcv.edu.mx (Ángel de Jesús Albino-Flores), ep.garciatejada@ugto.mx (Esthela Paola García-Tejada), e.diaz@ugto.mx (Erik Diaz-Cervantes), 


\subsection{Staphylococcus aureus}

Existen diferentes tipos de bacterias nosocomiales, aunque en este trabajo nos centraremos en Staphylococcus aureus por su capacidad para adquirir resistencia bacteriana rápidamente. $S$. aureus pertenece a la microbiota del ser humano, se aloja principalmente en la piel y mucosa, cuando estas barreras se rompen la bacteria puede alcanzar tejidos profundos y producir daños como: infecciones leves en la piel y tejidos blandos, endocarditis, neumonía, síndrome de choque tóxico, entre otras (Cervantes-García et al., 2014; McGuinness et al., 2017).

\subsection{Quinolonas}

Las quinolonas son una familia de antimicrobianos que se encarga de inhibir la enzima ADN-topoisomerasa principalmente en baterías gramnegativas y topoisomerasa IV en grampositivas para producir la inhibición bacteriana. Se clasifican en generaciones, las cuales se desarrollan a partir de la estructura de ácido nalidíxico (1-etil, 7-metil, 4-oxo, 1,8-naftiridina, 3-ácido carboxílico), esta y otras quinolonas de primera generación fueron usadas en el tratamiento de infecciones en el tracto urinario no complicadas (Sepúlveda et al., 2008).

En la segunda generación las quinolonas mejoran su actividad sobre bacterias gramnegativas mediante la adición de un átomo de flúor en la posición 6 y adquieren efecto sobre grampositivas con la adición de una piperazina en la posición 7 .

En la tercera generación se mejora el espectro de actividad de las generaciones predecesoras mediante nuevos sustituyentes como $-\mathrm{NH}_{2},-\mathrm{CH}_{3},-\mathrm{OH}$ que se enfocan en mejorar su efecto sobre grampositivas.

Finalmente, en la cuarta generación inicia cobertura contra microorganismos anaerobios con la adición de un átomo de $\mathrm{N}$ en la posición 8 (Álvarez-Hernández et al., 2015; Pham et al., 2019).

Las quinolonas se encargan de inhibir la enzima ADN girasa en bacterias gramnegativas y Topoisomerasa IV en grampositivas, ambas son enzimas tetraméricas compuestas por dos subunidades A, y dos subunidades B, codificadas por los genes gyrA (97 kDa) y gyrB $(90 \mathrm{kDa})$ en el caso de ADN girasa, y en Topoisomerasa IV son parC (75 kDa) y parE (70 kDa) (Alos, 2009; Jacoby, 2005).

En general, las Topoisomerasas II se encargan del superenrollamiento y desenrollamiento necesario para evitar daños en la estructura helicoidal durante la replicación y transcripción de ADN. Para lograr dicha tarea, estas enzimas rompen ambas cadenas de ADN con el objetivo pasar un segmento a través de otro y posteriormente religar las cadenas cortadas (Álvarez-Hernández et al., 2015; Chan et al., 2015). Las quinolonas se unen al $\mathrm{ADN}$ en el punto de corte mediante puentes de hidrógeno o interacciones de apilamiento, formando un complejo estable quinolona-ADN-Topoisomerasa que evita el religado de las cadenas de ADN. Al inhibir la acción de estas enzimas se evita la síntesis de proteínas necesarias para la reparación, crecimiento y reproducción bacteriana, lo que de forma prolongada conlleva a la muerte de la célula (RodríguezMartínez, 2005).

\subsection{Nano-acarreadores}

El metabolismo que generan algunos órganos como el intestino, hígado y riñones a los fármacos para su excreción es un obstáculo para proveer las concentraciones adecuadas. Se han desarrollado sistemas a partir de polímeros biocompatibles como polietilenglicol (Vashist et al., 2018) y quitosano (Garcia-Fuentes \& Alonso, 2012) y nanopartículas metálicas capaces de almacenar el fármaco y liberarlo en su sitio de acción en concentraciones controlables, con el objetivo de optimizar las concentraciones necesarias para presentar una actividad farmacológica eficaz y evitar los efectos adversos ocasionados por altas concentraciones.

Se ha reportado en literatura el uso de compositos de $\mathrm{TiO}_{2}$ y diseño de cúmulos con aplicación biológica (Aguilera-Granja et al., 2016), es por esto que en este trabajo se realiza un estudio preliminar con nano-acarreadores a base de titanio.

El objetivo de este trabajo es diseñar moléculas a partir de la estructura base de una quinolona y evaluar el efecto de triazoles, tetrazoles e isatinas comparando: la concentración mínima inhibitoria (MIC, por sus siglas en inglés), obtenida a través un modelo matemático predictivo; y la energía de acoplamiento a la enzima topoisomerasa IV de $S$. aureus (blando biológico de quinolonas), con la de quinolonas usadas comercialmente. Además, se realiza un estudio preliminar acerca de dióxido de titanio $\left(\mathrm{TiO}_{2}\right)$ como nano-acarreador de quinolonas para la entrega efectiva al blanco biológico.

\section{Metodología}

Se seleccionó un grupo de quinolonas de uso comercial con mayor actividad farmacológica y se recopilaron valores MIC reportados en literatura. De igual manera se seleccionó una estructura inicial basada en la celda unitaria de brookita de $\mathrm{TiO}_{2}$ (con un tamaño aproximado de $6.705 \AA ̊$ ), capeando las orillas con hidrógenos para evitar valencias libres.

Posteriormente se optimizó la geometría de estas estructuras mediante la Teoría de Funcionales de la Densidad (DFT, por sus siglas en inglés) utilizando el funcional/base M06-L/6-31G (d,p) para obtener las energías óptimas como sistema neutro y $6-311+\mathrm{G}$ $(\mathrm{d}, \mathrm{p})$ para sistemas en anión y catión, con el objetivo de obtener datos más precisos en el cálculo de descriptores.

\subsection{Estudio QSAR}

El estudio de relación cuantitativa estructura-actividad (QSAR, por sus siglas en inglés) se realizó con el programa MobyDigs, en el cual, un total de 1,246 descriptores moleculares, electrónicos y geométricos fueron analizados para formar ecuaciones lineales con aquellos descriptores que tengan mayor influencia en la variable seleccionada. Para seleccionar el modelo matemático final se realizó un análisis de componentes principales en el que se descartaron aquellos modelos que mostraron correlación entre sus variables.

\subsection{Acoplamientos moleculares}

Este estudio se realizó para observar el sitio de unión de quinolonas comerciales al blanco biológico para posteriormente, comparar los resultados con las estructuras diseñadas y compósitos de quinolona- $\mathrm{TiO}_{2}$. Como blanco biológico se utilizó la estructura tridimensional de la enzima Topoisomerasa IV de $S$. aureus, obtenida del Protein Data Bank bajo el código 5CDP; mientras que las quinolonas comerciales y estructuras diseñadas actuaron como ligando. Para los acoplamientos de Topoisomerasa IV con el compósito se realizó un acoplamiento previo quinolona$\mathrm{TiO}_{2}$

\section{Resultados}

\subsection{Estudio QSAR}

El modelo matemático obtenido se muestra a continuación (1): 
$0.11828 * S C B O-20.31352 * A R R-14.8091 *$
$M A T S 3 v+0.01453 * G(N . . O)-1.11867=p M I C$

Donde SCBO es la suma de enlaces (sin intervención de hidrógenos); ARR es la fracción de la molécula con anillos aromáticos; MATS3v es una autocorrelación de Moran en función a los volúmenes atómicos de van der Waals y; G(N..O) es la distancia geométrica entre átomos de nitrógeno y oxígeno. Se calculó un coeficiente de determinación $\left(\mathrm{R}^{2}\right)$ de 0.9727 , lo que nos indica una alta correlación entre el valor de referencia y el valor calculado.

En la Tabla 1 se muestran los valores MIC calculados a partir del modelo matemático seleccionado y los valores MIC experimentales de quinolonas comerciales. Se puede observar que las estructuras con mejor MIC calculado tienen un valor $>35$ del descriptor SCBO y $>78$ de G(N..O) lo que sugiere que estos son los valores recomendables para que una quinolona posea una actividad farmacológica de tercera o cuarta generación. En el caso de ARR y MATS3v aportan de manera negativa en la ecuación, por lo que a medida que disminuyen estos valores, obtendremos mejores valores MIC. Se establece un rango de entre -0.063 y 0.018 para el descriptor MTS3v, mientras que para ARR es necesario tener un valor $<0.210$.

Tabla 1: MIC calculado in silico y MIC experimental de quinolonas comerciales

\begin{tabular}{|c|c|c|c|c|c|c|c|c|}
\hline & & & & & & & In silico & experimental \\
\hline Estructura & SCBO & ARR & MATS3v & G(N..O) & pMIC & $\mathrm{MIC}(\mathrm{mg} / \mathrm{L})$ & pMIC & $\mathrm{MIC}(\mathrm{mg} / \mathrm{L})$ \\
\hline Cinoxacina & 27 & 0.286 & -0.086 & 45.311 & -1.803 & 63.508 & -1.806 & 64 \\
\hline $\begin{array}{c}\text { Ácido } \\
\text { oxolínico }\end{array}$ & 27 & 0.286 & -0.216 & 23.384 & -0.196 & 1.571 & -0.193 & 1.56 \\
\hline Norfloxacina & 32 & 0.240 & -0.076 & 68.705 & -0.085 & 1.217 & -0.079 & 1.20 \\
\hline Lomefloxacina & 34 & 0.222 & -0.036 & 68.319 & -0.081 & 1.205 & -0.079 & 1.20 \\
\hline Ciprofloxacina & 34 & 0.222 & -0.063 & 68.534 & 0.322 & 0.476 & 0.301 & 0.50 \\
\hline Levofloxacina & 36 & 0.207 & -0.024 & 78.953 & 0.437 & 0.365 & 0.602 & 0.25 \\
\hline Ofloxacina & 36 & 0.207 & -0.024 & 78.953 & 0.437 & 0.365 & 0.301 & 0.50 \\
\hline Gatifloxacina & 37 & 0.200 & -0.018 & 79.034 & 0.610 & 0.246 & 0.602 & 0.25 \\
\hline Esparfloxacina & 38 & 0.194 & -0.019 & 83.359 & 0.928 & 0.118 & 0.921 & 0.12 \\
\hline Trovafloxacina & 44 & 0.353 & -0.184 & 89.248 & 0.937 & 0.116 & 1.222 & 0.06 \\
\hline Clinafloxacina & 35 & 0.214 & -0.103 & 70.367 & 1.222 & 0.060 & 1.222 & 0.06 \\
\hline Moxifloxacina & 40 & 0.182 & -0.03 & 80.759 & 1.533 & 0.029 & 1.222 & 0.06 \\
\hline
\end{tabular}

\subsection{Diseño de estructuras}

Se construyó un andamio base a partir de las quinolonas comerciales más efectivas y se realizaron pruebas con grupos funcionales unidos al primer nitrógeno de los anillos de una quinolona. El andamio base utilizado se muestra en la Figura 1, en las primeras cuatro estructuras se cambió el grupo metoxilo por un átomo de $\mathrm{F}$ en la posición 5 .

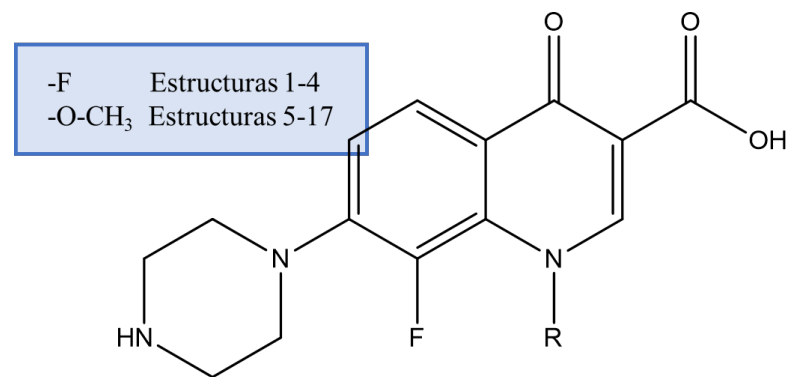

Figura 1. Andamio base para diseño de estructuras

En el primer grupo (estructuras 1-4) se utilizó un ciclopropil, ciclobutil, ciclohexano y 2,4 difluorofenilo cada uno de ellos saturados; en los grupos dos (5-10) y tres (11-15) se emplearon triazoles y tetrazoles respectivamente, variando la posición de los nitrógenos y número de insaturaciones; finalmente en el cuarto grupo se realizaron pruebas con una isatina unida al nitrógeno en la estructura 16 y unida al oxígeno en la 17 como se muestra en la Figura 2.

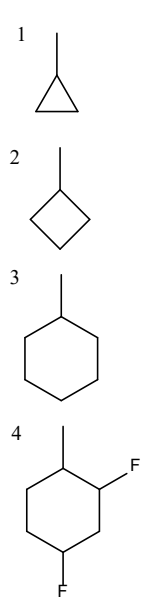<smiles>CN1CCNCN1</smiles><smiles>CN1CCCN=N1</smiles><smiles>CN1C(=O)C(=O)c2ccccc21</smiles><smiles>CN1CNCNN1C</smiles><smiles>CN1CC=CN=NN1C</smiles><smiles>COC1Nc2ccccc2C1=O</smiles>

Figura 2. Sustituyentes de estructuras propuestas

En la Tabla 2 se muestran los valores MIC calculados in silicco. Se observa que las estructuras propuestas alcanzaron valores MIC similares a las quinolonas comerciales, por ejemplo, el MIC de la estructura $16(0.25530 \mathrm{mg} / \mathrm{L})$ es comparable con el MIC de ciprofloxacina $(0.476 \mathrm{mg} / \mathrm{L})$ la cual es una quinolona usada en la actualidad.

Las estructuras 12, 7 y 11 obtuvieron el MIC más bajo de las estructuras propuestas, comparten la posición de nitrógenos 1,2 y 6 en el anillo del azol, esto nos indica que esta configuración resulta favorable en el efecto bactericida de una quinolona, probablemente debido a la atracción que experimentan los electrones de los átomos al flúor generando una densidad electrónica mayor que beneficia la formación de enlaces al blanco biológico. 
En algunas estructuras similares como la 7 y 12,10 y 15,9 y 14, 8 y 13 se puede observar que 3 átomos de nitrógeno en el anillo son suficientes para tener una buena actividad farmacológica, y al añadir otro átomo de nitrógeno puede resultar desfavorable, probablemente debido a una pérdida del equilibrio en la densidad electrónica al estar tan cerca del flúor.
Al comparar la configuración de las estructuras 10 y 9, 15 y 14 se puede observar que el número de insaturaciones mejora la acción farmacológica de la molécula, por la posible conjugación electrónica que influye en el apilamiento tipo stacking (apilamiento entre anillos).

Tabla 2: MIC calculado de estructuras propuestas

\begin{tabular}{ccccccc}
\hline & & & & & & In silico \\
\hline Estructura & SCBO & ARR & MATS3v & G(N..O) & pMIC & MIC (mg/L) \\
1 & 35 & 0.214 & -0.016 & 68.465 & -0.094 & 1.242285 \\
2 & 35 & 0.207 & -0.031 & 68.405 & 0.269 & 0.537975 \\
3 & 38 & 0.194 & -0.023 & 68.352 & 0.769 & 0.170251 \\
4 & 40 & 0.182 & -0.056 & 68.487 & 1.740 & 0.018201 \\
5 & 38 & 0.188 & -0.093 & 159.345 & 3.250 & 0.000563 \\
6 & 38 & 0.188 & -0.052 & 163.37 & 2.701 & 0.001991 \\
7 & 38 & 0.188 & -0.176 & 153.192 & 4.389 & 0.000041 \\
8 & 38 & 0.188 & -0.114 & 156.349 & 3.517 & 0.000304 \\
9 & 39 & 0.188 & -0.114 & 156.991 & 3.645 & 0.000227 \\
10 & 40 & 0.188 & -0.114 & 158.021 & 3.778 & 0.000167 \\
11 & 38 & 0.188 & -0.147 & 183.973 & 4.407 & 0.000039 \\
12 & 38 & 0.188 & -0.127 & 182.29 & 4.086 & 0.000082 \\
13 & 38 & 0.188 & -0.083 & 188.093 & 3.519 & 0.000303 \\
14 & 39 & 0.188 & -0.083 & 187.584 & 3.630 & 0.000234 \\
15 & 40 & 0.188 & -0.083 & 189.56 & 3.777 & 0.000167 \\
16 & 49 & 0.316 & -0.019 & 141.335 & 0.593 & 0.255301 \\
17 & 48 & 0.316 & -0.055 & 139.571 & 0.982 & 0.104192 \\
\hline
\end{tabular}

\subsection{Acoplamiento quinolona con Topoisomerasa IV}

Las quinolonas comerciales mostraron afinidad por el surco mayor de ADN (Figura 3). Se intercalaron paralelamente entre los anillos de las bases nitrogenadas para formar interacciones de apilamiento, puentes de hidrógeno con los nucleótidos y ocasionalmente mostraron interacciones estéricas con algunos aminoácidos de la enzima como serina (Ser) y ácido glutámico (Glu).

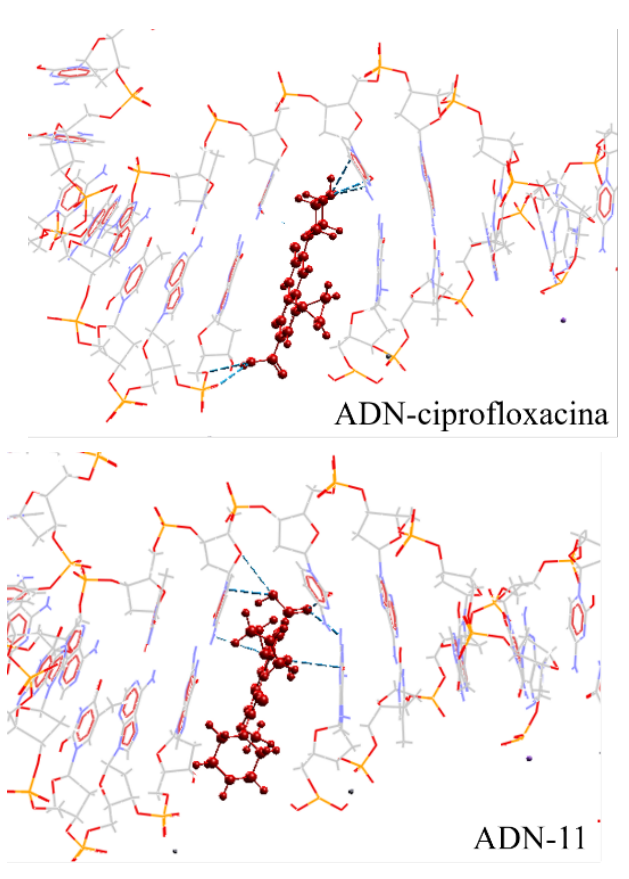

Figura 3. Sitio de unión de quinolonas y estructuras propuestas

Las estructuras propuestas también mostraron afinidad por el surco mayor de ADN, por lo que podrían tener el mismo mecanismo de acción. Además, la energía de acoplamiento es similar a la que mostraron las quinolonas comerciales. Por 
ejemplo, en el diseño 7 se calculó una energía de $-129.163 \mathrm{eV}$, mientras que lomefloxacina obtuvo $-129.941 \mathrm{eV}$. En algunos casos la energía de acoplamiento era incluso más estable en las estructuras propuestas, como las estructuras $16(-152.326 \mathrm{eV})$ y $17(-164.548 \mathrm{eV})$ en comparación con la energía de clinafloxacina $(-147.525 \mathrm{eV})$ y trovafloxacina $(-155.741 \mathrm{eV})$.

\subsection{Acoplamiento quinolona-TiO $\mathrm{T}_{2}$ con Topoisomerasa IV}

Los compósitos quinolona- $\mathrm{TiO}_{2}$ se situaron principalmente en los costados de la estructura helicoidal formando puentes de hidrógeno con nucleótidos e interacciones estéricas con aminoácidos de la enzima-blanco (Figura 4); aunque en algunas estructuras se presentó un acoplamiento en el surco mayor de ADN como pose secundaria. En la Tabla 3 se muestran algunas energías de acoplamiento de quinolonas y estructuras propuestas al estar unidas a una celda unitaria de $\mathrm{TiO}_{2}$. Se puede observar que la energía de unión disminuye al estar unidas a una celda unitaria de $\mathrm{TiO}_{2}$ debido a que algunos átomos de oxígeno contribuyen en la formación de puentes de hidrógeno con nucleótidos del $\mathrm{ADN}$, lo que resulta favorable para la entrega efectiva de fármacos.
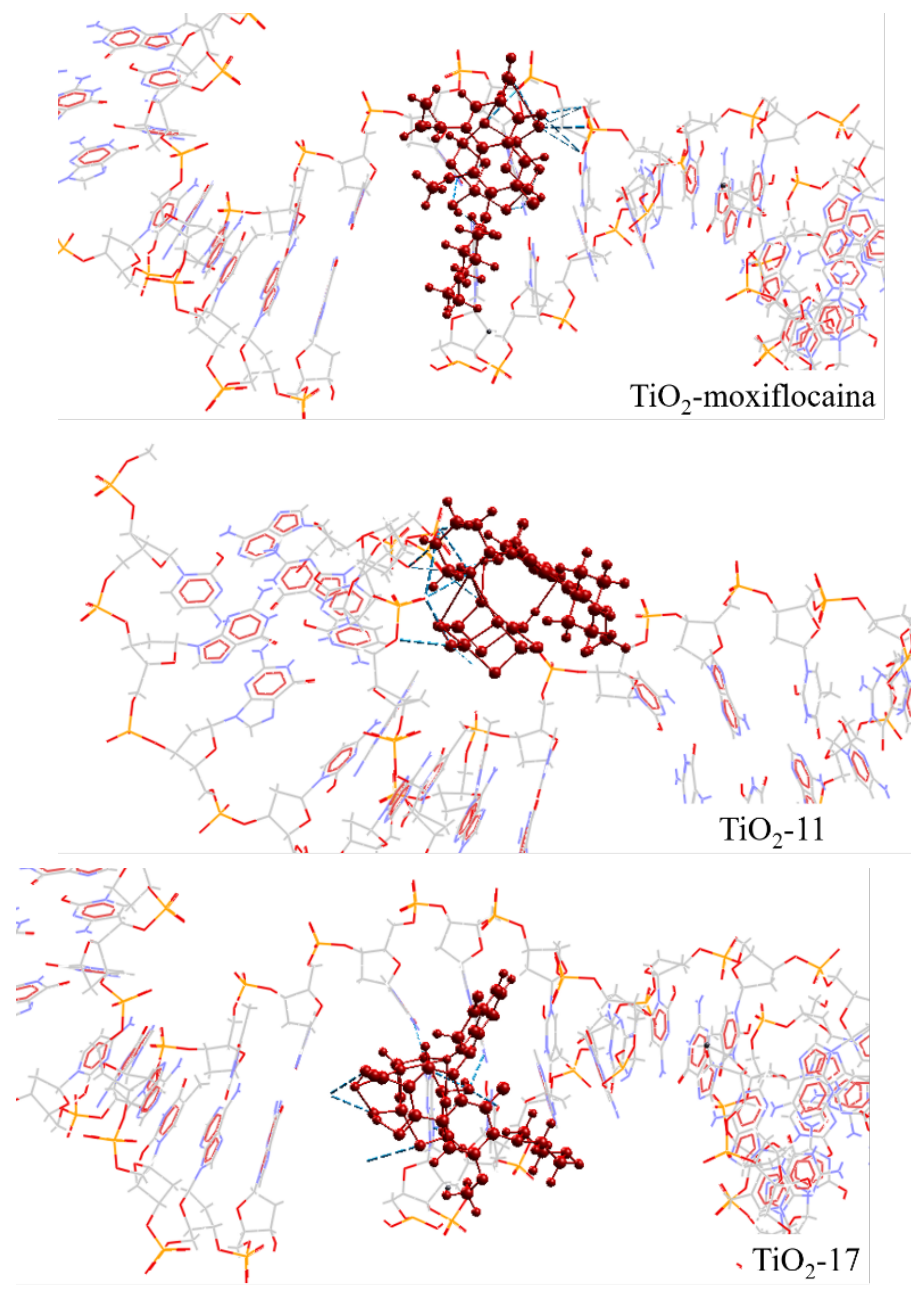

Figura 4. Sitio de unión de compósitos quinolona-TiO
Tabla 3: Energía de acoplamiento de quinolonas y compósito quinolona- $\mathrm{TiO}_{2}$ al blanco biológico.

\begin{tabular}{ccc}
\hline Estructura & $\begin{array}{c}\text { Acoplamiento ADN- } \\
\text { quinolona (eV) }\end{array}$ & $\begin{array}{c}\text { Acoplamiento ADN- } \\
\text { TiO }_{2} \text {-quinolona (eV) }\end{array}$ \\
\hline Ciprofloxacina & -134.091 & -149.208 \\
Moxifloxacina & -146.899 & -151.286 \\
11 & -135.840 & -146.408 \\
17 & -164.548 & -169.168 \\
\hline
\end{tabular}

\section{Conclusiones}

Se determinó que las estructuras diseñadas tendrían un mecanismo de inhibición similar al de las quinolonas comerciales.

Se hallaron y cuantificaron algunas características importantes para que una quinolona tenga una actividad bactericida similar a la de una quinolona de tercera o cuarta generación. Además, se determinó que el número de insaturaciones tiene un impacto positivo en la actividad farmacológica de una quinolona. El grupo funcional 1, 2, 6 triazol e isatina son los sustituyentes más destacados en este trabajo.

La estabilidad energética de acoplamiento al blanco biológico se ve favorecida al formar un compósito quinolona$\mathrm{TiO}_{2}$, situándose en los costados de la cadena de ADN. Por lo que es viable la entrega efectiva de quinolonas mediante un nano-acarreador de $\mathrm{TiO}_{2}$.

La química computacional es una alternativa viable en los primeros pasos del desarrollo de nuevos medicamentos. Permite estimar las propiedades biológicas de moléculas que aún no han sido sintetizadas, de este modo se pueden descartar y hallar estructuras prometedoras en el diseño de nuevos fármacos. También colabora en la comprensión del mecanismo de acción de diversos fármacos mediante modelado computacional.

\section{Agradecimientos}

En agradecimiento con el Laboratorio Nacional de Caracterización de Propiedades Fisicoquímicas y Estructura Molecular por el tiempo de cómputo provisto en el Clúster Pípila y al Laboratorio de Caracterización Molecular de Biosistemas y Nanocompuestos (LACAMBION).

\section{Referencias}

Aguilera-Granja, F., Vega, A., \& Balbás, L. C. (2016). New structural and electronic properties of $\left(\mathrm{TiO}_{2}\right)$ 10. The Journal of Chemical Physics, 144(23), 234312.

Alos, J.-I. (2009). Quinolonas. Enfermedades Infecciosas y Microbiologia Clinica, 27(5), 290-297.

Álvarez-Hernández, D. A., Garza-Mayén, G. S., \& Vazquez-Lopez, R. (2015). Quinolones. Nowadays perspectives and mechanisms of resistance. Revista Chilena de Infectologia: Organo Oficial de La Sociedad Chilena de Infectologia, 32(5), 499-504.

Cervantes-García, E., García-González, R., \& Salazar-Schettino, P. M. (2014). Características generales del Staphylococcus aureus. Revista Mexicana de Patología Clínica y Medicina de Laboratorio, 61(1), 28-40.

Chan, P. F., Srikannathasan, V., Huang, J., Cui, H., Fosberry, A. P., Gu, M., Hann, M. M., Hibbs, M., Homes, P., Ingraham, K., \& others. (2015). Structural basis of DNA gyrase inhibition by antibacterial QPT-1, anticancer drug etoposide and moxifloxacin. Nature Communications, 6(1), 1-13.

Garcia-Fuentes, M., \& Alonso, M. J. (2012). Chitosan-based drug 
nanocarriers: where do we stand? Journal of Controlled Release, 161(2), 496-504.

Jacoby, G. A. (2005). Mechanisms of resistance to quinolones. Clinical Infectious Diseases, 41(Supplement $\backslash 2)$, S120--S126.

Lebeque Pérez, Y., Morris Quevedo, H. J., \& Calás Viamonte, N. (2006). Infecciones nosocomiales: incidencia de la Pseudomonas aeruginosa. Revista Cubana de Medicina, 45(1), 0.

López-Cerero, L. (2014). Papel del ambiente hospitalario y los equipamientos en la transmisión de las infecciones nosocomiales. Enfermedades Infecciosas y Microbiología Clínica, 32(7), 459-464.

Maguiña Vargas, C. (2016). Infecciones nosocomiales. Acta Médica Peruana, 33(3), 175-177.

Mazón Sánchez, J. A., \& Verneiulle Román, J. S. (2019). Factores de riesgos que influyen en el desarrollo de las enfermedades nosocomiales.

McGuinness, W. A., Malachowa, N., \& DeLeo, F. R. (2017). Focus: infectious diseases: vancomycin resistance in Staphylococcus aureus. The Yale Journal of Biology and Medicine, 90(2), 269.
Oteo Iglesias, J., \& others. (2019). Comprendiendo la resistencia a antibióticos.

Pachay Solórzano, J. W. (2018). Las infecciones bacterianas y su resistencia a los antibióticos. Caso de estudio: Hospital Oncológico "Dr. Julio Villacreses Colmont Solca", Portoviejo. Revista Universidad y Sociedad, 10(5), 219-223.

Pham, T. D. M., Ziora, Z. M., \& Blaskovich, M. A. T. (2019). Quinolone antibiotics. Medchemcomm, 10(10), 1719-1739.

Rodríguez-Martínez, J. M. (2005). Mecanismos de resistencia a quinolonas mediada por plásmidos. Enfermedades Infecciosas y Microbiologia Clinica, 23(1), 25-31.

Sepúlveda, A. E. C., Enríquez, M. E. M., \& Patiño, N. M. (2008). Quinolonas. Revista de La Facultad de Medicina UNAM, 51(4), 173-177.

Vashist, A., Kaushik, A., Ghosal, A., Bala, J., Nikkhah-Moshaie, R., A Wani, W., Manickam, P., \& Nair, M. (2018). Nanocomposite hydrogels: advances in nanofillers used for nanomedicine. Gels, 4(3), 75. 